\title{
A novel three-miRNA signature predicts survival in cholangiocarcinoma based on RNA-Seq data
}

\author{
JUNYU CAO, LIANKANG SUN, JIE LI, CANCAN ZHOU, LIANG CHENG, KE CHEN, \\ BIN YAN, WEIKUN QIAN, QINGYONG MA and WANXING DUAN \\ Department of Hepatobiliary Surgery, The First Affiliated Hospital of Xi'an Jiaotong University, \\ Xi'an, Shaanxi 710061, P.R. China
}

Received January 1, 2018; Accepted May 22, 2018

DOI: $10.3892 / o r .2018 .6534$

\begin{abstract}
Accumulating evidence illustrates that many microRNAs (miRNAs) are abnormally expressed in cholangiocarcinoma and play important roles in tumorigenesis, tumor progression and metastasis. These miRNAs may serve as prognostic biomarkers and potential therapeutic targets. The aim of the present study was to identify the differentially expressed miRNAs in cholangiocarcinoma tissues vs. normal tissues by analyzing high-throughput data derived from The Cancer Genome Atlas (TCGA) database. Furthermore, we evaluated the prognostic performance of the differentially expressed miRNAs and developed a novel three-miRNA signature which predicted survival in cholangiocarcinoma patients. According to the cut-off criteria of $\mathrm{P}<0.01$ and $\mid \log _{2} \mathrm{FCl}>1.0$, a total of $100 \mathrm{miRNAs}$ (54 upregulated and 46 downregulated) were found to be differentially expressed and some of them were significantly associated with clinical features. Of the above 100 miRNAs, we obtained three miRNAs (miR-10b, miR-22 and miR-551b) which were markedly related to patient overall survival (OS). Subsequently, a novel three-miRNA signature was established and validated to be effective to predict survival. The results demonstrated that the survival rate, as well as the survival time of patients were obviously enhanced in relation to a lower miRNA signature index. Univariate and multivariate Cox regression analyses revealed that the three-miRNA signature was an independent prognostic factor in cholangiocarcinoma. The reliability of the three-miRNA signature was validated by an independent cohort from Gene Expression Omnibus (GEO). Furthermore, the functional enrichment analysis emphasized that the target
\end{abstract}

Correspondence to: Dr Wanxing Duan or Professor Qingyong Ma, Department of Hepatobiliary Surgery, The First Affiliated Hospital of Xi'an Jiaotong University, 277 West Yanta Road, Xi'an, Shaanxi 710061, P.R. China

E-mail: 0556029@fudan.edu.cn

E-mail: qyma56@xjtu.edu.cn

Key words: cholangiocarcinoma, miRNA signature, TCGA, overall survival, prognosis genes of the aforementioned miRNAs may be involved in a variety of pathways and processes associated with cancer. Finally, these three miRNAs were detected for verification of expression using RT-qPCR, and miR-551b was selected for the verification of biological functions in cholangiocarcinoma cells. The results revealed that overexpression of miR-551b decreased cancer cell proliferation and promoted apoptosis. Collectively, the results of the present study indicated that a specific three-miRNA signature could be considered as an alternative prognostic marker in cholangiocarcinoma.

\section{Introduction}

Cholangiocarcinoma, a highly aggressive tumor derived from bile duct epithelial cells, is one of the most severe forms of cancer with a 5 -year survival rate $<10 \%(1,2)$. During the last decades, the incidence and mortality rate of cholangiocarcinoma have been increasing globally $(3,4)$. The survival quality and prognosis of patients with cholangiocarcinoma are poor as a result of early cancer cell invasion and metastasis (2). Radical surgery is the only curative treatment for cholangiocarcinoma, while patients gain little benefit, as they are usually diagnosed at an advanced stage $(5,6)$. Thus, the exploration of powerful markers which may provide prognostic value for cholangiocarcinoma patients is of great significance. Currently, prognostic microRNA (miRNA) expression signatures in various cancers, such as colon cancer (7), clear cell renal cell carcinoma (8) and cervical cancer (9) have attracted the attention of researchers. Therefore, the miRNA signature has been regarded as an important change in cholangiocarcinoma progression and therapy (10).

miRNAs, a class of small non-coding RNAs of $\sim 22-23$ nucleotides in length, are considered to play pivotal roles in post-transcriptional gene regulation. It has been confirmed that miRNAs regulate malignancies by binding to the partially complementary recognition sequences in the 3-untranslated region of mRNAs, which causes target mRNA translation inhibition or degradation (11). A number of miRNAs play vital roles in tumorigenesis, such as cell proliferation, apoptosis, autophagy, migration, invasion and metastasis $(12,13)$. Accordingly, miRNAs have a large potential to serve as markers in the diagnosis, prognosis and targeted therapies of cancers. 
Although a large number of miRNAs have been identified in predicting the clinical outcome in cholangiocarcinoma, there are some limitations in previous studies. These may be due to molecular and clinical heterogeneity in different studies, relatively limited numbers of miRNAs, along with methodological differences in detection and analysis. The Cancer Genome Atlas (TCGA) is a National Cancer Institute effort to profile $>20$ different tumor types using genomic platforms and to make raw and processed data available to researchers worldwide (14). TCGA provides a collection of clinical data, RNA sequence, DNA copy number variations, DNA methylation and miRNA sequence profiles for cholangiocarcinoma. The aim of the present study was to identify the differentially expressed miRNAs between cholangiocarcinoma and normal tissues by analyzing high-throughput data downloaded from TCGA database. Furthermore, we evaluated the prognostic performance of the differentially expressed miRNAs and established a novel three-miRNA signature which could effectively predict survival in cholangiocarcinoma patients.

\section{Materials and methods}

TCGA dataset of cholangiocarcinoma. The miRNA sequencing data and corresponding clinical information for cholangiocarcinoma patients (up to June 29, 2017) were downloaded from TCGA data portal (https://portal.gdc.cancer. gov). The inclusion criteria were set as follows: i) samples with both miRNA sequencing data and clinical information; and ii) samples with detailed prognostic information. Finally, a total of 45 samples were enrolled in this study, including 36 cholangiocarcinoma tissues and 9 matched normal tissues.

Exploration of the differentially expressed miRNAs in cholangiocarcinoma. The RNA-Seq data of cholangiocarcinoma with 1,046 miRNAs were analyzed on the Illumina HiSeq miRNA Seq platform. Subsequently, the R language package 'edgeR' was used for the calculation of differentially expressed miRNAs (15). The expression difference of individual miRNAs was characterized by $\log _{2} \mathrm{FC}$ and adjusted P-value. LogFC indicates the fold change in expression of each miRNA between cholangiocarcinoma tissues and normal tissues. Upregulated and downregulated miRNAs were determined based on $\log _{2} \mathrm{FC}>1$ and $\log _{2} \mathrm{FC}<-1$ respectively, with adjusted $\mathrm{P}<0.01$. The miRNAs which had expression mean value $<1$ were excluded.

Selection of the cut-offpoint for the Kaplan-Meier survival analysis. Cutoff Finder (http://molpath.charite.de/cutoff) was used to determine a cut-off point for patient stratification into two groups (16). Then, the differences in patient overall survival (OS) between the high-level and the low-level group were evaluated by Kaplan-Meier survival analysis (log-rank method). The miRNAs with a P-value $<0.01$ were regarded to display statistically significant differences between groups and were considered for further analysis.

Association between miRNA signature index and OS. A value of one or zero was assigned to patients according to each miRNA value. Subsequently, each miRNA value was scored in the signature. Thus, each patient would have a score, defined as miRNA signature index. We set index as high-risk and low-risk into two new groups according to the index value. Then, Kaplan-Meier survival analysis (log-rank method) was performed to evaluate the differences in patient OS between these two groups.

Information collection of the validation dataset. An independent cohort of cholangiocarcinoma patients (GSE53870) (17) downloaded from Gene Expression Omnibus (GEO) database was used for the prognostic signature validation. There consisted of 63 cholangiocarcinoma patients and corresponding prognostic information in the GSE53870 dataset.

Target gene prediction of three prognostic miRNAs and functional analysis. The target genes of the three prognostic miRNAs were predicted using TargetScan (http://www. targetscan.org/), miRDB (http://www.mirdb.org/miRDB/), and miRanda (http://www.microrna.org/) online analysis tools. To further increase the bioinformatics analysis reliability, Venn diagram was carried out to identify the overlapping target genes. Furthermore, the Gene Ontology (GO) annotation and Kyoto Encyclopedia of Genes and Genomes (KEGG) pathway enrichment analysis of target genes were performed using the Database for Annotation, Visualization and Integrated Discovery (DAVID) online tool (https://david.ncifcrf.gov/). The results were then presented in a bubble diagram.

$R N A$ isolation and $q R T-q P C R$. Total RNA was extracted from cells using an HP Total RNA kit (Omega Biotech, Stamford, CT, USA) according to the manufacturer's protocol. Synthesis of cDNA with reverse transcriptase (RT) was performed with an M-MLV First Strand kit (Life Technologies; Thermo Fisher Scientific, Inc., Gaithersburg, MD, USA). Primer sequences for miR-10b, miR-22, miR-551b and U6 detection were obtained from RiboBio (Guangzhou, China). The RT primers for mature miRNAs and U6 were designed according to the concept of a stem-loop RT primer (18). RT-qPCR analysis was carried out using Platinum SYBR-Green qPCR SuperMix-UDG kits (Life Technologies) according to the manufacturer's protocol. Real-time PCR was performed on an Applied Biosystems ABI PRISM 7500 Real-Time PCR system (Thermo Fisher Scientific, Inc.). Ct values of miRNAs were equilibrated to U6, which was used as an internal control. Relative expression was calculated using the $2^{-\Delta \Delta \mathrm{Cq}}$ method.

Cell culture and transfection. Human cholangiocarcinoma cell line HUCCT1 (cat. no. JCRB0425) was purchased from the Japanese Collection of Research Bioresources Cell Bank (JCRB; Osaka, Japan) and human intrahepatic biliary epithelial cell line (HiBEC) (cat. no. 5100) was purchased from the ScienCell Research Laboratories (San Diego, CA, USA) and were cultured under standard conditions. When HUCCT1 cells reached 50-70\% confluence, miR-551b mimics and negative control were transfected using Invitrogen ${ }^{\mathrm{TM}}$ Lipofectamine 2000 (Thermo Fisher Scientific, Inc., Waltham, MA, USA) according to the manufacturer's protocol. miR-551b mimics (sense, 5'-GCGACCCAUACUUGGUUUCAG-3' and antisense, 5'-GAAACCAAGUAUGGGUCGCUU-3') and corresponding negative control were purchased from GenePharma (Shanghai, China). 
Proliferation assay. HUCCT1 cells were transfected with miR-551b mimics or negative control for $48 \mathrm{~h}$, and then were plated into 96-well plates at a density of $5 \times 10^{3}$ cells/well and then $10 \mu 1$ of 3-(4,5-dimethyl-2-thiazolyl)-2,5-diphenyl-2Htetrazolium bromide (MTT, $5 \mathrm{mg} / \mathrm{ml}$; Sigma-Aldrich; Merck KGaA, Darmstadt, Germany) was added and incubated for $4 \mathrm{~h}$. The supernatant was then replaced with $100 \mu \mathrm{l}$ of dimethyl sulfoxide (DMSO; Sigma-Aldrich; Merck KGaA) and read at $490 \mathrm{~nm}$ using a multifunction microplate reader (POLARstar OPTIMA; BMG, Offenburg, Germany). Concerning the colony formation assay, HUCCT1 cells $\left(1 \times 10^{3}\right.$ cells/dish) were seeded on $35-\mathrm{mm}$ petri dishes. Cells were further cultured for two weeks to allow colonies to form. At the indicated time-point, colonies were fixed with $4 \%$ paraformaldehyde, stained with $0.1 \%$ crystal violet solution and rinsed. Then, images were captured using a Nikon camera (Nikon Corp., Tokyo, Japan) and the colony number was counted.

Apoptosis assay. HUCCT1 cells were transfected with miR-551b mimics or negative control for $48 \mathrm{~h}$, then were harvested by trypsinization in a tube and were washed twice in ice-cold phosphate-buffered saline (PBS). After staining using an Annexin V-FITC/7-AAD apoptosis detection kit (Becton-Dickinson, Franklin Lakes, NJ, USA) according to the manufacturer's protocol, the cell apoptosis rate was assessed using a FACSCalibur flow cytometer (BD Biosciences, San Diego, CA, USA).

Statistical analysis. Mann-Whitney U test was used to compare the expression levels of miRNAs between two different groups of each clinical characteristic. Univariate/multivariate Cox proportional hazard regression analyses were performed to compare each clinical parameter and prognostic miRNA signature (high-risk vs. low-risk). All statistical analysis was performed by SPSS 20.0 (SPSS, Inc., Chicago, IL, USA). Statistical significance was defined as a two-sided $\mathrm{P}$-value $<0.05$, unless specifically indicated.

\section{Results}

Exploration of differentially expressed miRNAs. A total of 45 samples were enrolled in our study, including 36 cholangiocarcinoma tissues and 9 matched normal tissues. The specific clinical characteristics included sex, age at diagnosis, stage, $\mathrm{T}$ stage, lymph node status, metastasis and histological type (Table I). According to the cut-off criteria of $\mathrm{P}<0.01$ and $\mid \log _{2} \mathrm{FCl}>1.0$, a total of $100 \mathrm{miRNAs}$ were found to be differentially expressed in cholangiocarcinoma vs. normal tissues, including 54 upregulated and 46 downregulated miRNAs (Table II). In order to show the above differentially expressed miRNA more clearly, we present the results as a Volcano plot (Fig. 1). Furthermore, it was obvious that cholangiocarcinoma tissues could be clearly discriminated from normal tissues in terms of differentially expressed miRNA patterns using unsupervised hierarchic cluster analysis (Fig. 2).

Association between differentially expressed miRNAs and clinical features. The differentially expressed miRNAs were further analyzed upon the expression level and clinical
Table I. Clinical characteristics of the cholangiocarcinoma patients $(n=36)$.

\begin{tabular}{lr}
\hline Variables & Cases, $\mathrm{n}(\%)$ \\
\hline Sex & \\
Female & $20(55.56)$ \\
Male & $16(44.44)$ \\
Age at diagnosis (years) & \\
$\leq 60$ & $14(38.89)$ \\
$>60$ & $22(61.11)$ \\
Stage & \\
I+II & $28(77.78)$ \\
III+IV & $8(22.22)$ \\
T stage & \\
T1+T2 & $31(86.11)$ \\
T3+T4 & $5(13.89)$ \\
Lymph node status & \\
N0 & $26(72.22)$ \\
N1 & $5(13.89)$ \\
NX & $5(13.89)$ \\
Metastasis & \\
M0 & $28(77.78)$ \\
M1 & $5(13.89)$ \\
MX & $3(8.33)$ \\
Histological type & \\
Intrahepatic & $30(83.33)$ \\
Hilar+distal & $6(16.67)$ \\
\hline
\end{tabular}

characteristics. Notably, miR-490 and miR-141 were found to be related with sex, whereas miR-615, miR-135b, miR-92b, miR-23c and miR-149 were found to be related with age at diagnosis. Furthermore, miR-301a was associated with stage, whereas miR-551b, miR-222 and miR-221 were associated with $\mathrm{T}$ stage, miR-92b and miR-615 were associated with lymph node status, miR-101-1 and miR-301a were associated with metastasis. In addition, we also found other 8 miRNAs which were linked to histological type (Table III).

Identification of three miRNAs associated with OS. Of the aforementioned 100 miRNAs, we used Cutoff Finder to determine a cut-off point and classified patients into two groups based on the miRNA expression level. Subsequently, the Kaplan-Meier survival analysis was performed to explore the association between miRNA expression and OS in cholangiocarcinoma patients. According to the survival analysis, three miRNAs were identified to be significantly associated with OS in cholangiocarcinoma patients. These three miRNAs were miR-10b, miR-22 and miR-551b. These results illustrated that high expression levels of miR-10b and miR-551b were considered as better prognostic markers vs. the low level group (Fig. 3A and C). In contrast, compared to the high-level group, low expression level of miR-22 revealed a longer survival rate and time (Fig. 3B). 
Table II. Differentially expressed miRNAs between cholangiocarcinoma and normal tissues.

\begin{tabular}{|c|c|c|c|c|c|c|c|}
\hline \multicolumn{4}{|c|}{ Upregulated miRNAs } & \multicolumn{4}{|c|}{ Downregulated miRNAs } \\
\hline miRNAs & $\log \mathrm{FC}$ & P-value & FDR & miRNAs & $\log \mathrm{FC}$ & P-value & FDR \\
\hline hsa-mir-182 & 4.50 & $1.72 \mathrm{E}-26$ & 8.03E-24 & hsa-mir-148a & -2.67 & $1.12 \mathrm{E}-25$ & $2.61 \mathrm{E}-23$ \\
\hline hsa-mir-183 & 4.85 & $2.93 \mathrm{E}-25$ & $4.56 \mathrm{E}-23$ & hsa-mir-1258 & -4.77 & $3.35 \mathrm{E}-20$ & $3.91 \mathrm{E}-18$ \\
\hline hsa-mir-96 & 4.77 & $5.87 \mathrm{E}-19$ & $3.92 \mathrm{E}-17$ & hsa-mir-378 & -2.93 & $2.32 \mathrm{E}-19$ & $2.17 \mathrm{E}-17$ \\
\hline hsa-mir-21 & 2.53 & $1.09 \mathrm{E}-16$ & $4.26 \mathrm{E}-15$ & hsa-mir-101-1 & -2.02 & $3.13 \mathrm{E}-19$ & $2.44 \mathrm{E}-17$ \\
\hline hsa-mir-27a & 1.93 & $2.97 \mathrm{E}-12$ & $8.15 \mathrm{E}-11$ & hsa-let-7c & -2.10 & $1.39 \mathrm{E}-18$ & $8.10 \mathrm{E}-17$ \\
\hline hsa-mir-34c & 4.83 & 3.39E-11 & 7.92E-10 & hsa-mir-99a & -2.28 & $2.15 \mathrm{E}-18$ & $1.11 \mathrm{E}-16$ \\
\hline hsa-mir-200b & 2.80 & 8.91E-11 & 1.89E-09 & hsa-mir-505 & -2.02 & $2.44 \mathrm{E}-18$ & $1.14 \mathrm{E}-16$ \\
\hline hsa-mir-92b & 2.94 & $9.35 \mathrm{E}-11$ & $1.90 \mathrm{E}-09$ & hsa-mir-139 & -2.76 & $4.65 \mathrm{E}-18$ & $1.97 \mathrm{E}-16$ \\
\hline hsa-mir-200a & 2.67 & $1.05 \mathrm{E}-09$ & $2.04 \mathrm{E}-08$ & hsa-mir-125b-2 & -2.25 & $5.65 \mathrm{E}-16$ & $2.03 \mathrm{E}-14$ \\
\hline hsa-mir-34b & 6.26 & $1.28 \mathrm{E}-09$ & 2.39E-08 & hsa-mir-378c & -2.72 & $1.15 \mathrm{E}-14$ & 3.83E-13 \\
\hline hsa-mir-181d & 2.54 & 2.29E-09 & $3.97 \mathrm{E}-08$ & hsa-mir-490 & -4.27 & $4.53 \mathrm{E}-14$ & $1.41 \mathrm{E}-12$ \\
\hline hsa-mir-23a & 1.49 & $1.07 \mathrm{E}-08$ & $1.49 \mathrm{E}-07$ & hsa-mir-675 & -4.08 & $1.80 \mathrm{E}-13$ & $5.25 \mathrm{E}-12$ \\
\hline hsa-mir-222 & 1.98 & $1.26 \mathrm{E}-08$ & $1.69 \mathrm{E}-07$ & hsa-mir-1468 & -2.20 & $1.10 \mathrm{E}-11$ & $2.84 \mathrm{E}-10$ \\
\hline hsa-mir-181b-1 & 2.11 & $1.83 \mathrm{E}-08$ & $2.37 \mathrm{E}-07$ & hsa-mir-483 & -3.40 & $1.85 \mathrm{E}-11$ & $4.55 \mathrm{E}-10$ \\
\hline hsa-mir-429 & 2.75 & $3.28 \mathrm{E}-08$ & 4.14E-07 & hsa-mir-101-2 & -1.80 & $8.32 \mathrm{E}-11$ & 1.85E-09 \\
\hline hsa-mir-454 & 1.70 & $3.62 \mathrm{E}-08$ & $4.45 \mathrm{E}-07$ & hsa-mir-424 & -1.63 & $1.46 \mathrm{E}-09$ & 2.62E-08 \\
\hline hsa-mir-221 & 1.82 & $5.94 \mathrm{E}-08$ & $6.93 \mathrm{E}-07$ & hsa-mir-122 & -4.09 & $2.42 \mathrm{E}-09$ & 4.04E-08 \\
\hline hsa-mir-330 & 1.76 & 7.15E-08 & $8.14 \mathrm{E}-07$ & hsa-mir-885 & -3.80 & $3.21 \mathrm{E}-09$ & 5.18E-08 \\
\hline hsa-mir-135b & 4.88 & $2.04 \mathrm{E}-07$ & $2.11 \mathrm{E}-06$ & hsa-mir-22 & -1.27 & $5.24 \mathrm{E}-09$ & $8.15 \mathrm{E}-08$ \\
\hline hsa-let-7e & 1.36 & $3.31 \mathrm{E}-07$ & $3.36 \mathrm{E}-06$ & hsa-mir-383 & -2.81 & $6.81 \mathrm{E}-09$ & $1.02 \mathrm{E}-07$ \\
\hline hsa-mir-181c & 1.83 & 4.60E-07 & $4.57 \mathrm{E}-06$ & hsa-mir-551b & -2.54 & 7.01E-09 & $1.02 \mathrm{E}-07$ \\
\hline hsa-mir-708 & 2.62 & $2.01 \mathrm{E}-06$ & $1.77 \mathrm{E}-05$ & hsa-mir-574 & -1.36 & $1.09 \mathrm{E}-08$ & $1.49 \mathrm{E}-07$ \\
\hline hsa-mir-99b & 1.26 & 7.07E-06 & $5.88 \mathrm{E}-05$ & hsa-mir-192 & -1.91 & $4.51 \mathrm{E}-08$ & $5.40 \mathrm{E}-07$ \\
\hline hsa-mir-181b-2 & 2.00 & 7.17E-06 & $5.88 \mathrm{E}-05$ & hsa-mir-624 & -1.48 & 8.71E-08 & 9.69E-07 \\
\hline hsa-mir-301a & 1.27 & $1.39 \mathrm{E}-05$ & 0.000108 & hsa-mir-455 & -1.71 & $1.62 \mathrm{E}-07$ & $1.76 \mathrm{E}-06$ \\
\hline hsa-mir-181a-1 & 1.36 & $1.91 \mathrm{E}-05$ & 0.000144 & hsa-mir-152 & -1.17 & $1.82 \mathrm{E}-07$ & $1.93 \mathrm{E}-06$ \\
\hline hsa-mir-1301 & 1.50 & 2.29E-05 & 0.00017 & hsa-mir-194-2 & -1.67 & 4.97E-07 & $4.84 \mathrm{E}-06$ \\
\hline hsa-mir-196b & 4.30 & $2.38 \mathrm{E}-05$ & 0.000174 & hsa-mir-194-1 & -1.65 & 7.29E-07 & $6.95 \mathrm{E}-06$ \\
\hline hsa-mir-1266 & 2.18 & $2.70 \mathrm{E}-05$ & 0.000194 & hsa-mir-618 & -1.96 & $1.08 \mathrm{E}-06$ & $1.01 \mathrm{E}-05$ \\
\hline hsa-mir-561 & 3.32 & $2.91 \mathrm{E}-05$ & 0.000206 & hsa-mir-144 & -2.25 & $1.25 \mathrm{E}-06$ & $1.14 \mathrm{E}-05$ \\
\hline hsa-mir-200c & 5.13 & $3.64 \mathrm{E}-05$ & 0.000254 & hsa-mir-1228 & -1.55 & $1.38 \mathrm{E}-06$ & $1.24 \mathrm{E}-05$ \\
\hline hsa-mir-218-2 & 1.70 & $4.09 \mathrm{E}-05$ & 0.000277 & hsa-mir-511-2 & -1.69 & $3.88 \mathrm{E}-06$ & $3.35 \mathrm{E}-05$ \\
\hline hsa-mir-149 & 1.89 & $7.53 \mathrm{E}-05$ & 0.000488 & hsa-mir-511-1 & -1.73 & 4.10E-06 & $3.48 \mathrm{E}-05$ \\
\hline hsa-mir-141 & 4.73 & $9.43 \mathrm{E}-05$ & 0.000603 & hsa-mir-125b-1 & -1.17 & 7.87E-06 & $6.34 \mathrm{E}-05$ \\
\hline hsa-mir-625 & 1.69 & 0.00011 & 0.000683 & hsa-mir-548b & -2.35 & $1.28 \mathrm{E}-05$ & 0.000102 \\
\hline hsa-mir-10b & 2.38 & 0.000126 & 0.000774 & hsa-mir-3614 & -1.31 & $3.91 \mathrm{E}-05$ & 0.000269 \\
\hline hsa-mir-196a-1 & 5.03 & 0.000151 & 0.000883 & hsa-mir-33b & -1.48 & $6.25 \mathrm{E}-05$ & 0.000417 \\
\hline hsa-mir-1224 & 4.63 & 0.000177 & 0.001009 & hsa-mir-3648 & -1.79 & $7.18 \mathrm{E}-05$ & 0.000472 \\
\hline hsa-mir-3934 & 2.08 & 0.000189 & 0.00105 & hsa-mir-486 & -1.64 & 0.000107 & 0.000672 \\
\hline hsa-mir-615 & 5.88 & 0.000218 & 0.001184 & hsa-mir-23c & -1.46 & 0.000151 & 0.000883 \\
\hline hsa-mir-598 & 1.37 & 0.000332 & 0.00176 & hsa-mir-548j & -1.48 & 0.000184 & 0.001033 \\
\hline hsa-mir-187 & 2.69 & 0.000544 & 0.002704 & hsa-mir-328 & -1.06 & 0.000206 & 0.001135 \\
\hline hsa-mir-3200 & 1.82 & 0.000662 & 0.003252 & hsa-mir-365-1 & -1.03 & 0.000687 & 0.003343 \\
\hline hsa-mir-320b-2 & 1.66 & 0.00071 & 0.00342 & hsa-mir-211 & -2.16 & 0.001105 & 0.005009 \\
\hline hsa-mir-1180 & 1.15 & 0.000753 & 0.003587 & hsa-mir-451 & -1.44 & 0.001677 & 0.007107 \\
\hline hsa-mir-526b & 6.47 & 0.000834 & 0.003895 & hsa-mir-3944 & -1.78 & 0.002201 & 0.00871 \\
\hline hsa-mir-1304 & 2.47 & 0.000859 & 0.003972 & & & & \\
\hline hsa-mir-31 & 4.13 & 0.001327 & 0.005901 & & & & \\
\hline hsa-mir-3691 & 2.94 & 0.001532 & 0.006748 & & & & \\
\hline hsa-mir-218-1 & 2.09 & 0.001665 & 0.007107 & & & & \\
\hline
\end{tabular}


Table II. Continued.

\begin{tabular}{|c|c|c|c|c|c|c|c|}
\hline \multicolumn{4}{|c|}{ Upregulated miRNAs } & \multicolumn{4}{|c|}{ Downregulated miRNAs } \\
\hline miRNAs & $\log \mathrm{FC}$ & P-value & FDR & miRNAs & $\log \mathrm{FC}$ & P-value & FDR \\
\hline hsa-mir-577 & 3.92 & 0.001689 & 0.007107 & & & & \\
\hline hsa-mir-137 & 4.94 & 0.001893 & 0.007687 & & & & \\
\hline hsa-mir-196a-2 & 4.62 & 0.002038 & 0.008205 & & & & \\
\hline hsa-mir-30d & 1.13 & 0.00219 & 0.00871 & & & & \\
\hline
\end{tabular}

FDR, false discovery rate.

Table III. Differentially expressed miRNAs associated with clinical features.

\begin{tabular}{|c|c|c|}
\hline Variables & Upregulated miRNAs identified in TCGA & Downregulated miRNAs identified in TCGA \\
\hline $\begin{array}{l}\text { Sex } \\
\text { (female vs. male) }\end{array}$ & $\operatorname{miR}-141$ & $\operatorname{miR}-490$ \\
\hline $\begin{array}{l}\text { Age at diagnosis } \\
(\leq 60 \text { vs. }>60)\end{array}$ & miR-615, miR-135b, miR-92b, miR-149 & $\operatorname{miR}-23 c$ \\
\hline $\begin{array}{l}\text { Stage } \\
(\mathrm{I}+\mathrm{II} \text { vs. III+IV) }\end{array}$ & miR-301a & \\
\hline $\begin{array}{l}\text { T stage } \\
(\mathrm{T} 1+\mathrm{T} 2 \text { vs. } \mathrm{T} 3+\mathrm{T} 4)\end{array}$ & miR-222, miR-221 & $\operatorname{miR}-551 b$ \\
\hline $\begin{array}{l}\text { Lymph node status } \\
\text { (N0 vs. N1) }\end{array}$ & miR-92b, miR-615 & \\
\hline $\begin{array}{l}\text { Metastasis } \\
\text { (M0 vs. M1) } \\
\text { Histological type } \\
\text { (intrahepatic vs. hilar+distal) }\end{array}$ & $\begin{array}{l}\operatorname{miR}-301 a \\
\operatorname{miR}-23 a, \text { miR-196a-1, miR-27a, } \\
\text { miR-598, miR-31, mir-181c }\end{array}$ & $\begin{array}{l}\operatorname{miR}-101-1 \\
\text { miR-365-1, miR-383 }\end{array}$ \\
\hline
\end{tabular}

Definition of three-miRNA signature index for cholangiocarcinoma prognosis. In order to establish the miRNA signature index, we assigned a score for each patient. To be specific, patients who belonged to the shorter survival group received one score for each miRNA, while those who belonged to the longer survival group received a 0 score for each miRNA. Subsequently, we calculated the total score for each patient. Subsequently, we calculated the score for each patient. According to these criteria, the highest score was 3 and the lowest score was 0 . Subsequently, we ranked 36 cholangiocarcinoma patients based on their miRNA signature index and divided them into two new groups (Table IV). The high-risk group miRNA signature index score was 2-3, while the score in the low-risk group was 0-1. Markedly, Kaplan-Meier survival analysis illustrated that these two groups were significantly associated with patient OS (Fig. 4). In the low-risk group, $\sim 80 \%$ patients showed 5-year survival, while none of the patients survived $>5$ years in the high-risk group. Furthermore, the median survival of the low-risk group was markedly longer than that of the high-risk group (63.75 vs. 14.63 months). As a result, our findings demonstrated that the survival rate, as well as the survival time of patients were obviously enhanced in relation to a lower miRNA signature index.

Taking into account the following clinical parameters: Sex, age at diagnosis, stage, $\mathrm{T}$ stage, lymph node status, metastasis and histological type, univariate and multivariate Cox regression analysis was used to test the effect of the three-miRNA signature (high-risk vs. low-risk) on OS. In univariate analysis, stage [hazard ratio $(\mathrm{HR})=3.104, \mathrm{P}=0.040$ ] and three-miRNA signature $(\mathrm{HR}=6.013, \mathrm{P}<0.0001)$ were associated with $\mathrm{OS}$ in cholangiocarcinoma patients. In multivariate analysis, three-miRNA signature $(\mathrm{HR}=6.124, \mathrm{P}<0.0001)$ was revealed to be an independent prognostic factor in cholangiocarcinoma patients (Table V).

Three-miRNA signature verification in the validation cohort. In order to validate the performance of the prognostic miRNA signature, it was tested on the GSE53870 dataset derived from GEO database. As displayed in Fig. 5, miR-22 and miR-551b were observed to be markedly associated with OS in cholangiocarcinoma patients $(\mathrm{P}<0.05)$, while miR-10b was found to be marginally significant with patient $\mathrm{OS}(\mathrm{P}=0.0511)$. 


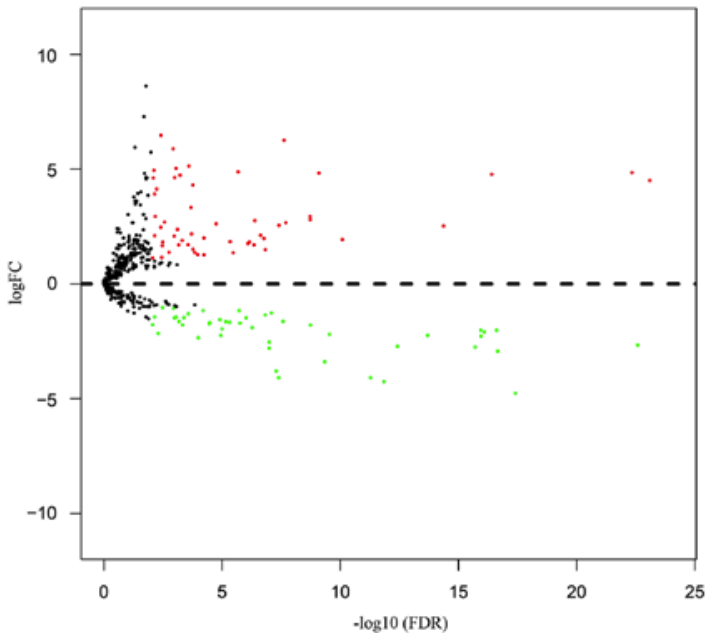

Figure 1. Volcano plot of differentially expressed miRNAs. The red dots represent upregulated miRNAs, and the green dots represent downregulated miRNAs. miRNAs, microRNAs; FDR, false discovery rate.

Subsequently, we used the same risk-score formula to calculate three-miRNA signature index for each of the 63 patients and dichotomized them into low-risk and high-risk group. Notably, these two groups were surprisingly associated with patient OS.
Based on the three-miRNA signature index, $>40 \%$ patients in the low-risk group showed 5-year survival, while none of the patients survived $>5$ years in the high-risk group. In addition, the median survival of the low-risk group was significantly longer than that of high-risk group (34.20 vs. 12.80 months). These findings were similar to what was noted in TCGA data. Collectively, these results proved the accuracy of the prognostic miRNAs signature.

KEGG pathway enrichment and GO annotation of three miRNA predicted genes. TargetScan, miRDB and miRanda online analysis tools were used to predict the target genes of these three miRNAs (miR-10b, miR-22 and miR-551b). A total of 110 overlapping genes of miR-10b, 222 overlapping genes of miR-22, and 2 overlapping genes of miR-551b were identified (Fig. 6). Then, enrichment analysis was performed to elucidate the biological function of consensus target genes. Notably, cancer-related pathways were found to be intensely activated according to KEGG results, including small cell lung cancer, chronic myeloid leukemia, prostate cancer, glioma, miRNAs in cancer and proteoglycans in cancer. We hypothesized that these target genes play pivotal roles in various types of cancers. Furthermore, target genes were significantly enriched in the mTOR, FoxO and HIF-1 signaling
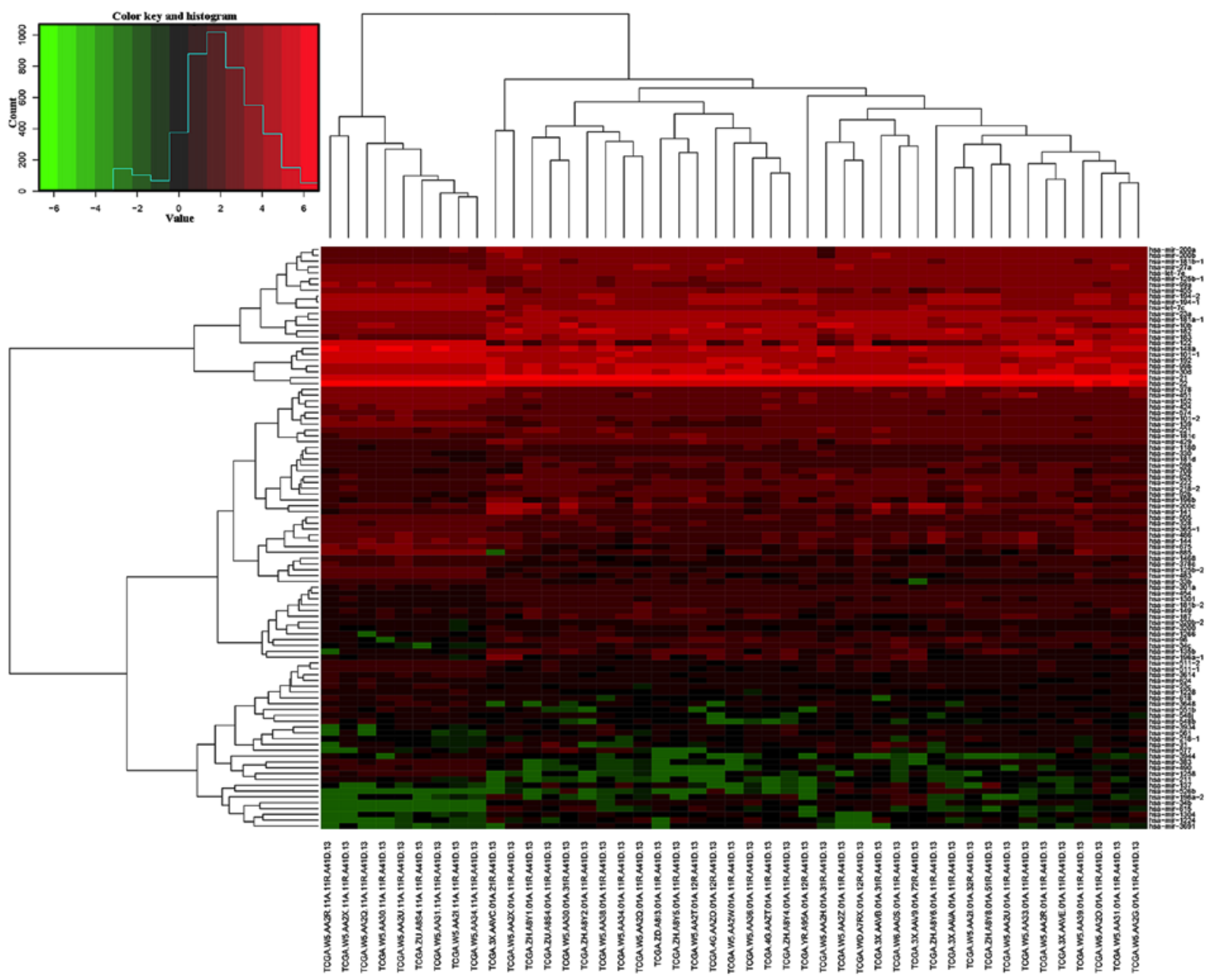

Figure 2. Hierarchical clustering of cancer tissues and non-cancer tissues by differentially expressed miRNAs. The heatmap consist of 9 normal tissues (left panel) and 36 cholangiocarcinoma tissues (right panel). Each row represents the expression level of a miRNA, and each column represents a sample. miRNAs, microRNAs. 
Table IV. Three-miRNA signature index for survival analysis.

\begin{tabular}{|c|c|c|c|c|c|c|}
\hline Patient ID & Survival status & Overall survival (months) & $\operatorname{miR}-10 b$ & miR-22 & $\mathrm{miR}-551 \mathrm{~b}$ & miRNA index \\
\hline TCGA-3X-AAVB & Alive & 13.22 & 0 & 0 & 0 & 0 \\
\hline TCGA-3X-AAVE & Alive & 21.37 & 0 & 0 & 0 & 0 \\
\hline TCGA-3X-AAVC & Alive & 23.31 & 0 & 0 & 0 & 0 \\
\hline TCGA-W5-AA2H & Alive & 35.41 & 0 & 0 & 0 & 0 \\
\hline TCGA-W5-AA33 & Alive & 47.64 & 0 & 0 & 0 & 0 \\
\hline TCGA-W5-AA2R & Alive & 50.70 & 0 & 0 & 0 & 0 \\
\hline TCGA-W5-AA2Q & Alive & 1.64 & 0 & 0 & 1 & 1 \\
\hline TCGA-4G-AAZT & Alive & 13.81 & 0 & 0 & 1 & 1 \\
\hline TCGA-ZH-A8Y8 & Alive & 19.79 & 0 & 0 & 1 & 1 \\
\hline TCGA-ZH-A8Y4 & Dead & 24.36 & 0 & 0 & 1 & 1 \\
\hline TCGA-W5-AA30 & Alive & 37.91 & 0 & 0 & 1 & 1 \\
\hline TCGA-4G-AAZO & Alive & 38.70 & 0 & 0 & 1 & 1 \\
\hline TCGA-ZH-A8Y5 & Alive & 40.41 & 0 & 0 & 1 & 1 \\
\hline TCGA-W5-AA36 & Dead & 46.09 & 1 & 0 & 0 & 1 \\
\hline TCGA-W5-AA38 & Alive & 48.36 & 0 & 0 & 1 & 1 \\
\hline TCGA-W5-AA2Z & Alive & 53.06 & 0 & 0 & 1 & 1 \\
\hline TCGA-W5-AA2I & Dead & 63.75 & 1 & 0 & 0 & 1 \\
\hline TCGA-W5-AA2G & Alive & 64.96 & 1 & 0 & 0 & 1 \\
\hline TCGA-W5-AA31 & Alive & 0.33 & 1 & 1 & 0 & 2 \\
\hline TCGA-WD-A7RX & Dead & 0.69 & 1 & 0 & 1 & 2 \\
\hline TCGA-ZU-A8S4 & Dead & 3.22 & 0 & 1 & 1 & 2 \\
\hline TCGA-ZD-A8I3 & Dead & 5.56 & 0 & 1 & 1 & 2 \\
\hline TCGA-W5-AA2X & Dead & 8.91 & 1 & 0 & 1 & 2 \\
\hline TCGA-3X-AAV9 & Dead & 11.15 & 0 & 1 & 1 & 2 \\
\hline TCGA-ZH-A8Y1 & Dead & 12.66 & 1 & 0 & 1 & 2 \\
\hline TCGA-W5-AA34 & Dead & 18.25 & 1 & 0 & 1 & 2 \\
\hline TCGA-W5-AA2U & Dead & 20.61 & 0 & 1 & 1 & 2 \\
\hline TCGA-W5-AA2O & Dead & 21.04 & 1 & 0 & 1 & 2 \\
\hline TCGA-ZH-A8Y2 & Dead & 23.05 & 1 & 0 & 1 & 2 \\
\hline TCGA-W6-AA0S & Alive & 26.56 & 0 & 1 & 1 & 2 \\
\hline TCGA-W5-AA2W & Dead & 30.38 & 1 & 0 & 1 & 2 \\
\hline TCGA-W5-AA2T & Dead & 40.11 & 1 & 0 & 1 & 2 \\
\hline TCGA-YR-A95A & Dead & 0.85 & 1 & 1 & 1 & 3 \\
\hline TCGA-W5-AA39 & Dead & 5.59 & 1 & 1 & 1 & 3 \\
\hline TCGA-3X-AAVA & Dead & 14.63 & 1 & 1 & 1 & 3 \\
\hline TCGA-ZH-A8Y6 & Alive & 17.06 & 1 & 1 & 1 & 3 \\
\hline
\end{tabular}

TCGA, The Cancer Genome Atlas.

A

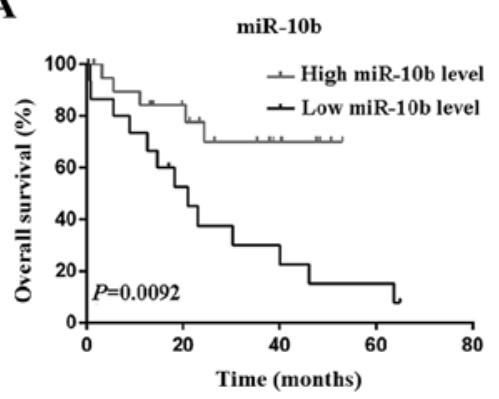

B

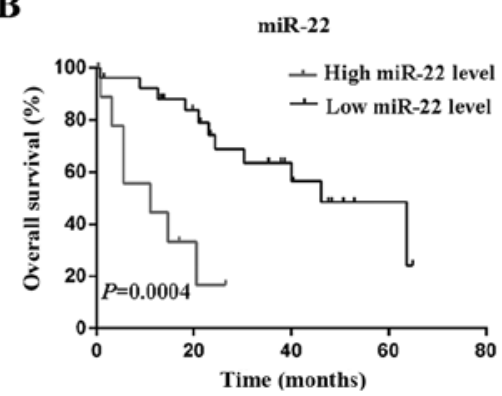

C

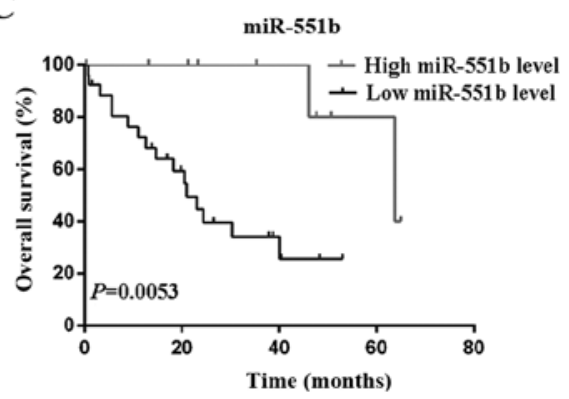

Figure 3. Kaplan-Meier survival curves for three miRNAs, (A) miR-10b, (B) miR-22 and (C) miR-551b, associated with OS in cholangiocarcinoma patients miRNAs, microRNAs; OS, overall survival. 
Table V. Univariate and multivariate analyses of parameters associated with overall survival.

\begin{tabular}{|c|c|c|c|c|}
\hline \multirow[b]{2}{*}{ Variables } & \multicolumn{2}{|c|}{ Univariate analysis } & \multicolumn{2}{|c|}{ Multivariate analysis } \\
\hline & HR $(95 \%$ CI $)$ & P-value & $\mathrm{HR}(95 \% \mathrm{CI})$ & P-value \\
\hline \multicolumn{5}{|l|}{ Sex } \\
\hline (male vs. female) & $1.387(0.544-3.534)$ & 0.494 & & \\
\hline \multicolumn{5}{|l|}{ Age at diagnosis } \\
\hline$(>60$ vs. $\leq 60)$ & $0.911(0.350-2.375)$ & 0.849 & & \\
\hline \multicolumn{5}{|l|}{ Stage } \\
\hline (III+IV vs. I+II) & 3.104 (1.051-9.169) & 0.040 & $2.983(0.981-9.072)$ & 0.054 \\
\hline \multicolumn{5}{|l|}{ T stage } \\
\hline (T3+T4 vs. T1+T2) & $0.660(0.150-2.896)$ & 0.582 & & \\
\hline \multicolumn{5}{|l|}{ Lymph node status } \\
\hline (N1 vs. N0) & $2.289(0.602-8.700)$ & 0.224 & & \\
\hline \multicolumn{5}{|l|}{ Metastasis } \\
\hline (M1 vs. M0) & $1.650(0.462-5.891)$ & 0.440 & & \\
\hline \multicolumn{5}{|l|}{ Histological type } \\
\hline (hilar+distal vs. intrahepatic) & $1.197(0.343-4.172)$ & 0.778 & & \\
\hline \multicolumn{5}{|l|}{ Three-miRNA signature } \\
\hline
\end{tabular}

HR, hazard ratio; CI, confidence interval.

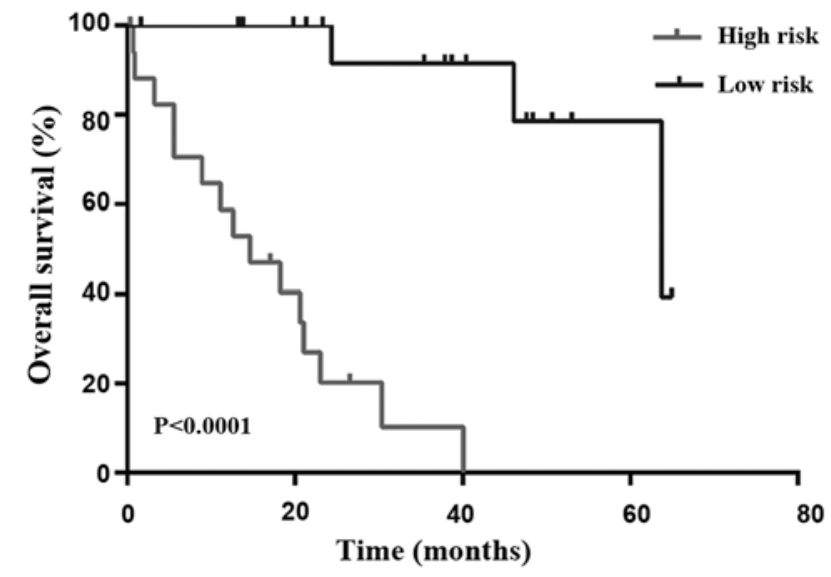

Figure 4. Kaplan-Meier survival curve for the three-miRNA signature in cholangiocarcinoma patients. miRNAs, microRNAs.

pathways (Fig. 7A). The GO biological process terms were mainly enriched in the regulation of metabolism, modification and transcription (Fig. 7B), which indicated that these three miRNAs may be closely associated with biological function and gene expression.

Effect of miR-551b overexpression on the proliferation and apoptosis in HUCCT1 cells. To further validate this conclusion, we first detected the expression of three miRNAs by RT-qPCR. According to the data in Fig. 8, two of the three tested miRNAs (miR-22 and miR-551b) yielded results quite similar to those of the TCGA data. Although the performance of miR-10b was not well-repeated, the direction of change was similar to that noted in the TCGA data. These results indicated that the differentially expressed miRNAs we identified by RNA-Seq data were reliable. As miR-551b is the least studied member among these three miRNAs in cancer, we here focused on its biological function in cholangiocarcinoma. miR-551b mimics were transfected into HUCCT1 cells to upregulate the expression of miR-551b. Successful overexpression of miR-551b in HUCCT1 cells was confirmed by RT-qPCR (Fig. 9A). MTT and colony formation assays revealed that overexpression of miR-551b significantly inhibited proliferation (Fig. 9B) and colony formation in HUCCT1 cells (Fig. 9C). In addition, flow cytometry analysis showed that overexpression of miR-551b significantly induced apoptosis in the HUCCT1 cells (Fig. 9D).

\section{Discussion}

In the last decade, miRNAs, as the master modulators of multiple biological and pathological processes, are a 'hot' research topic in the field of cancer development. miRNAs are regarded as a novel group of disease biomarkers for the stability and universality in human tissues (19). Currently, growing investigations have demonstrated specific miRNA profiles in multiple cancers, emphasizing the pivotal roles of miRNAs in the initiation and progression of cancer, including cholangiocarcinoma. Previous studies have demonstrated that many miRNAs are crucial for the initiation, progression, and metastasis of cholangiocarcinoma by regulating various processes, including cancer cell proliferation, apoptosis, 
A

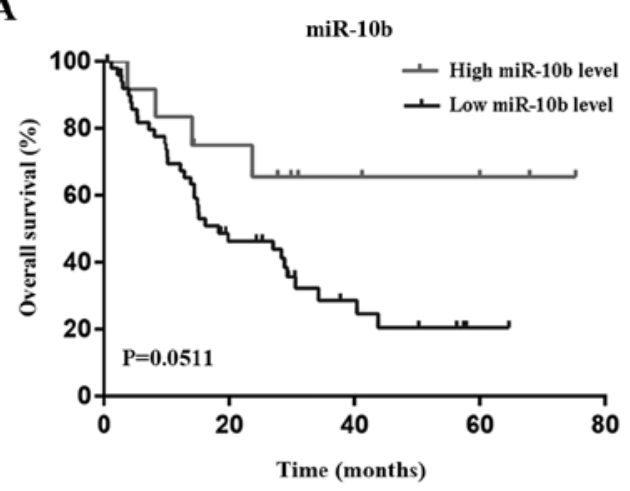

C

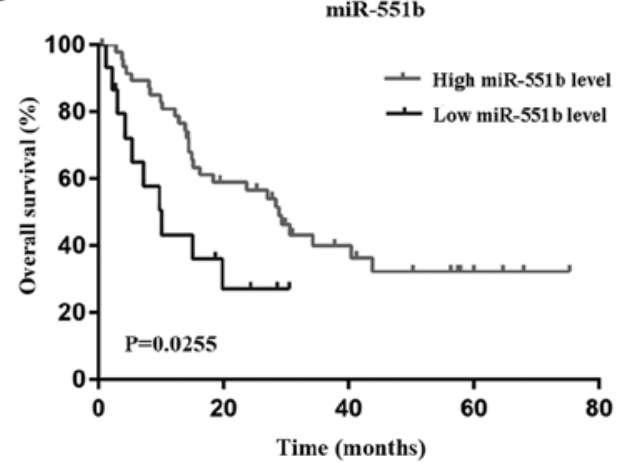

B

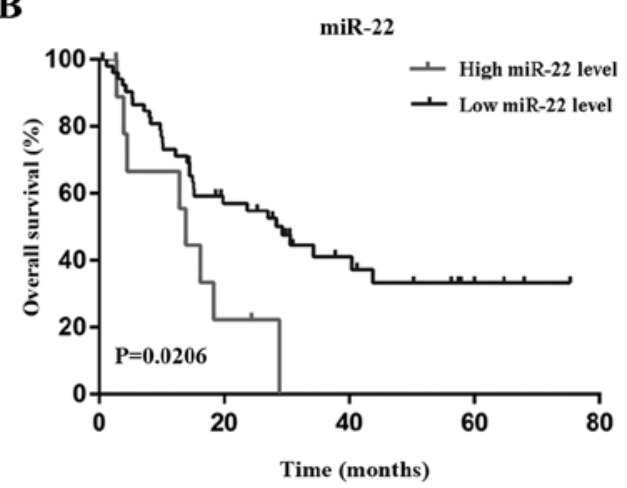

D

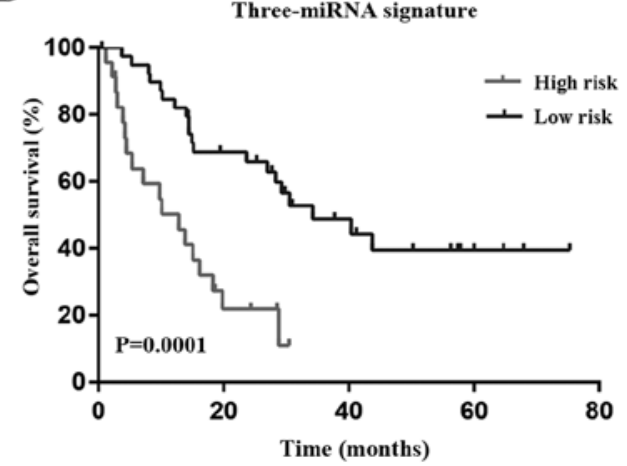

Figure 5. Validations of three miRNAs and three-miRNA signature in an independent cohort. (A) miR-10b was found to be marginally significant with patient OS ( $\mathrm{P}=0.0511)$. (B and $\mathrm{C}$ ) miR-22 and miR-551b were markedly associated with OS in cholangiocarcinoma patients ( $<<0.05)$. (D) Based on the three-miRNA signature index, $>40 \%$ patients in the low-risk group showed 5 -year survival, while none of the patients survived $>5$ years in the high-risk group. In addition, the median survival of the low-risk group was significantly longer than that of high-risk group (34.20 vs. 12.80 months) miRNAs, microRNAs; OS, overall survival.

A

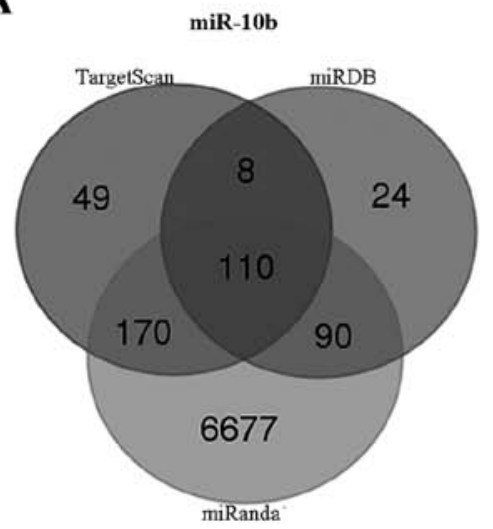

B

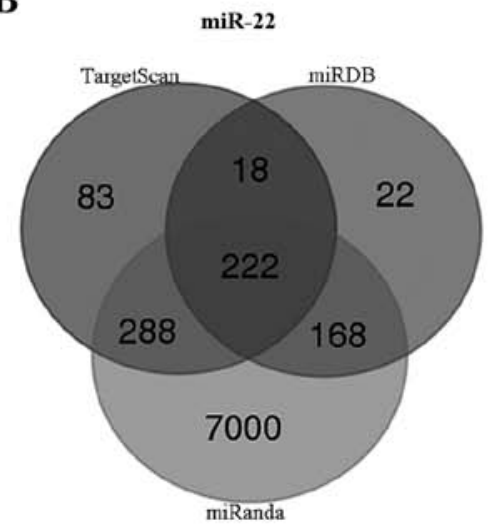

C

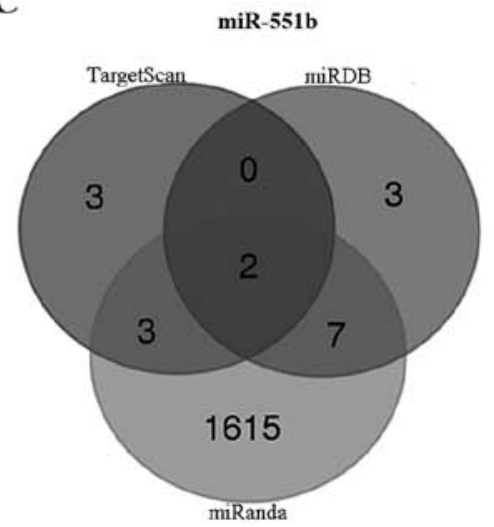

Figure 6. Target gene prediction of three prognostic miRNAs. The overlapping target genes of the three miRNAs, (A) miR-10b, (B) miR-22 and (C) miR-551b were predicted using TargetScan, miRDB and miRanda online analysis tools. miRNAs, microRNAs.

adhesion, cell cycle arrest, migration and invasion (20-23). To date, several studies have identified a number of miRNAs with prognostic value in cholangiocarcinoma, such as miR-126 (24), miR-192 (25), miR-26a (26), miR-203 (27), miR-106a (28) and miR-29a (29). Unfortunately, due to molecular and clinical heterogeneity in different studies, the relatively limited numbers of miRNAs to investigate, as well as the methodological differences in detection and analysis, there still exist some restrictions for applying the above specific miRNAs for prognosis.
TCGA was constructed to contain a wide assortment of high-throughput experimental data, which are available and will be valuable to researchers worldwide. In our study, we analyzed high-throughput data downloaded from TCGA database, and eventually obtained 100 differentially expressed miRNAs between cholangiocarcinoma and normal tissues, of which 54 were upregulated and 46 were downregulated. Subsequently, we evaluated the prognostic value of each differentially expressed miRNA. According to previous research, the performance of a single biomarker in predicting 
A

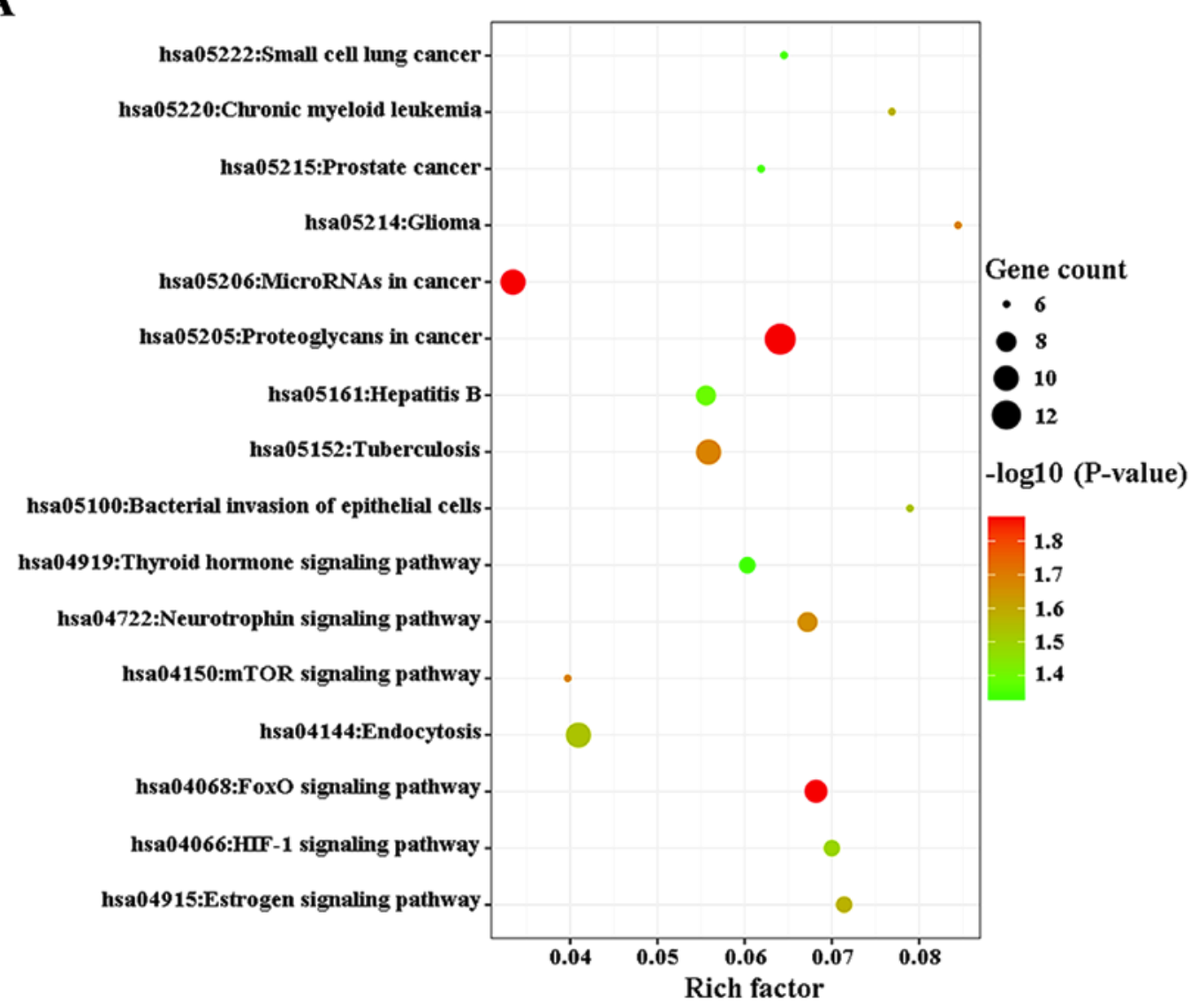

B

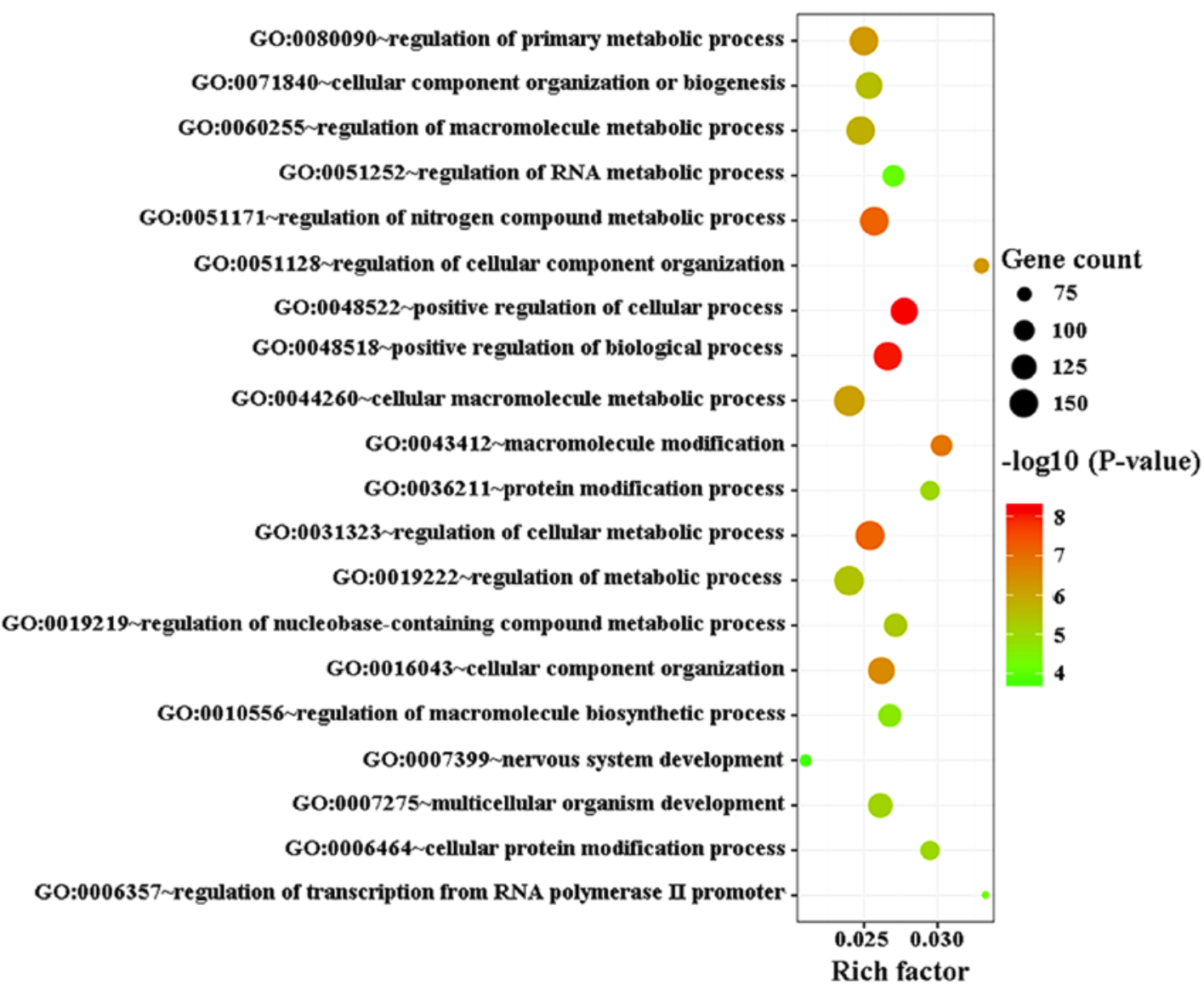

Figure 7. Functional analysis of target genes. (A) The significant enriched KEGG pathways of target genes. (B) The significant enriched GO biological processes of target genes. KEGG, Kyoto Encyclopedia of Genes and Genomes; GO Gene ontology. 


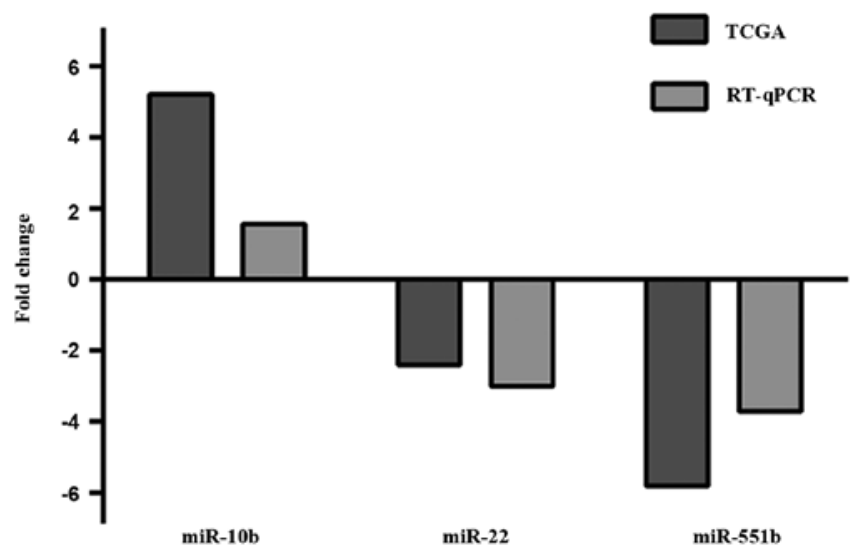

Figure 8. RT-qPCR validation of three miRNAs. Comparison of fold change of miRNAs between TCGA and RT-qPCR results. miRNAs, microRNAs; TCGA, The Cancer Genome Atlas.

survival across the datasets is unstable, while the combination of biomarkers increases the performance (30). Therefore, we established a novel three-miRNA signature (high-risk vs. low-risk) with excellent prognostic performance for cholangiocarcinoma patients. Although no statistically significant associations were observed between our three-miRNA signature and other clinical parameters (data not shown), it was then identified to be an independent prognostic factor and was successfully validated in an independent cohort from the GEO database.

Emerging evidence has demonstrated that numerous miRNAs are aberrantly expressed (upregulated or downregulated) in various cancers (31). Markedly, miRNAs show differential effects in multiple cancers, that is, they serve not only as tumor suppressors, but also as oncogenic promoters to hinder or aggravate cancer formation and malignant transformation. miR-10b, first reported as an oncogene in breast cancer, was found to induce the invasion and metastasis of breast cancer cells (32). Notably, a previous study also suggested that miR-10b may be a tumor suppressor in patients with gastric cancer and the lower level of miR-10b was detected in advanced stage small-cell carcinoma of the cervix patients compared to the early ones (33). Furthermore, miR-22, located on chromosome 17p13.3 (34), was reported to retard cellular growth, invasion and metastasis in cervical and
A

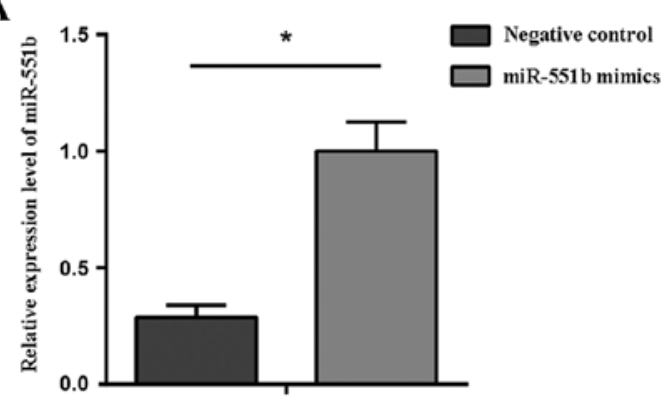

C
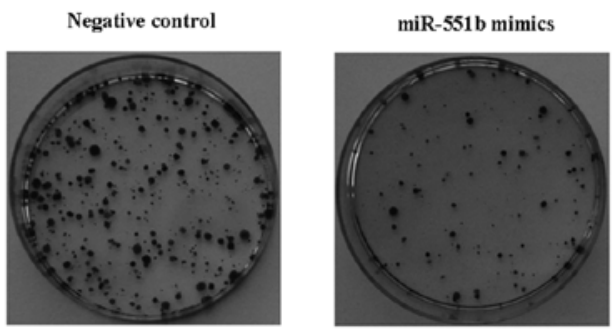

D

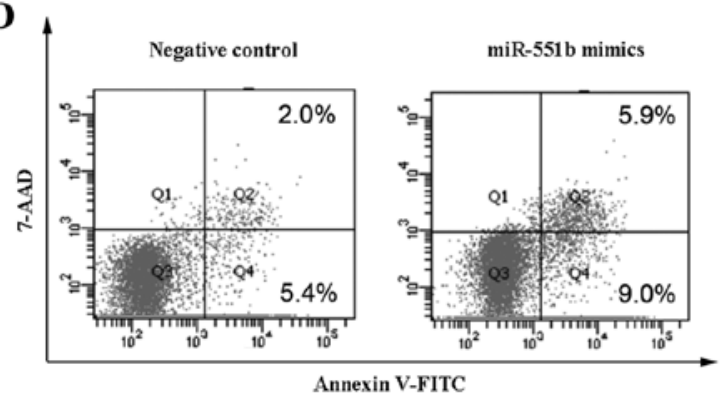

B
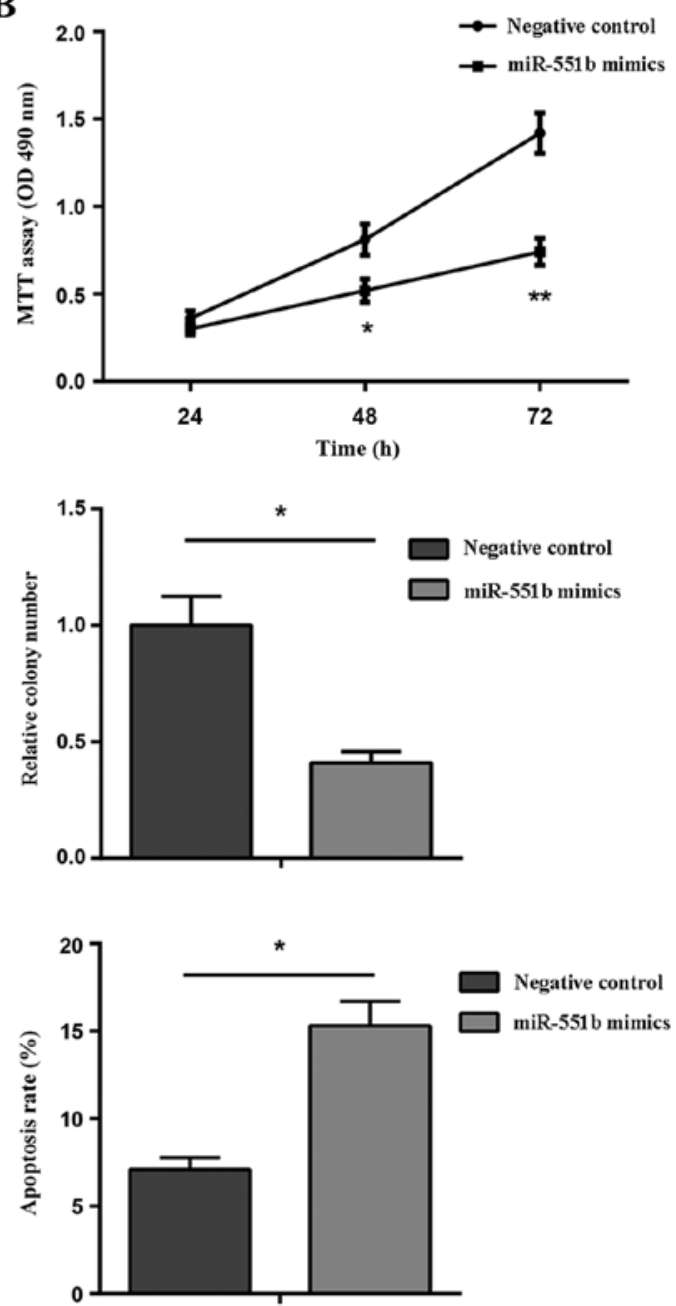

Figure 9. Overexpression of miR-551b inhibits proliferation and induces apoptosis in cholangiocarcinoma cells. (A) Overexpression of miR-551b using miR-551b mimics in cholangiocarcinoma cells. (B) Cell proliferation was determined in cholangiocarcinoma cells transfected with miR-551b mimics or negative control. (C) Cell colony formation was determined in cholangiocarcinoma cells transfected with miR-551b mimics or negative control. (D) Cell apoptosis was determined in cholangiocarcinoma cells transfected with miR-551b mimics or negative control. ${ }^{*} \mathrm{P}<0.05,{ }^{* *} \mathrm{P}<0.01$. 
breast cancer through inducing p53 expression and concurrently targeting SIRT1, CDK6 as well as Sp1 to activate $\mathrm{pRb}$ signaling pathway (35). However, Budd et al (36) reported that miR-22 in prostate cancer was overexpressed and promoted prostate cancer tumorigenesis by directly targeting PTEN. As for miR-551b, Lin et al (37) reported that it was upregulated in lung adenocarcinoma tissues compared to normal tissues and high level of miR-551b predicted a longer survival. Conversely, Song et al (38) found that miR-551b was downregulated in gastric cancer and suppressed EMT and metastasis in gastric cancer by inhibiting ERBB4. Furthermore, the functions of these three miRNAs in cholangiocarcinoma were poorly investigated. The expression and clinical information have provided us some clues to investigate the roles of these miRNAs in cholangiocarcinoma. Hence, we explored the biological functions of miR-551b in HUCCT1 cells and found that miR-551b overexpression inhibited proliferation and induced apoptosis, which was similar to that noted in Yuan et al recent study of gastric cancer (39).

To gain a deep insight into the molecular mechanisms of these three miRNAs, we predicted their target genes and analyzed the related pathways and GO annotations. Abnormal signaling pathways play crucial roles in the pathogenesis and progression of cholangiocarcinoma. We found that three miRNAs regulated several key signaling pathways, including mTOR, FoxO and HIF-1 signaling pathway. It has been well acknowledged that the PI3K/Akt/mTOR signaling pathway plays an important role in cholangiocarcinoma, and inhibition of mTOR kinase activity may be a viable approach for future application in patients with cholangiocarcinoma (40). FoxO transcription factors have been reported to play vital roles in tumorigenesis and drug resistance. Guan et al (41) reported that FoxO3 inactivation promoted human cholangiocarcinoma tumorigenesis and chemoresistance through Keap1-Nrf2 signaling. As for hypoxia inducible factor-1 (HIF-1), a family of heterodimeric proteins which includes HIF-1 $\alpha$ and HIF-1 $\beta$ subunits, has been identified to play an important role in initiation and progression of multiple cancers. Thongchot et al (42) reported that positive expression of HIF-1 $\alpha$ enhanced metastasis and predicted a poor prognosis of cholangiocarcinoma. Therefore, further molecular investigations are needed to confirm these predictions, and may provide new therapeutic interventions in cholangiocarcinoma.

However, there are some limitations in interpreting the above results. Firstly, a larger sample size was required to validate our findings. Secondly, the miRNA expression profiles were detected from bile duct tissues, which may not accurately reflect the levels of miRNAs in saliva, serum, urine or stool. Hence, we may need to explore the miRNAs signature in the above-mentioned samples since they are conveniently available for monitoring.

In conclusion, through performing an integrative analysis for differentially expressed miRNAs accompanied with relevant clinical data, we established a novel three-miRNA signature as an alternative prognostic predictor for cholangiocarcinoma patients. Further investigations are required to validate our findings and further functional studies are also needed to explore the potential molecular mechanisms of these miRNAs in cholangiocarcinoma.

\section{Acknowledgements}

Not applicable.

\section{Funding}

The present study was supported by grants from the National Natural Scientific Foundation of China (nos. 81402971, 81472248 and 81672434).

\section{Availability of data and materials}

The datasets used during the present study are available from the corresponding author upon reasonable request.

\section{Authors' contributions}

JC, QM and WD designed the study. JC, LS and JL collected the data. JC performed the bioinformatical analysis. CZ and LC performed the statistical analysis. JC wrote the main manuscript and prepared all figures. $\mathrm{KC}, \mathrm{BY}$ and $\mathrm{WQ}$ contributed to the revision of the manuscript and were also involved in the conception of the study. All authors read and approved the manuscript and agree to be accountable for all aspects of the research in ensuring that the accuracy or integrity of any part of the work are appropriately investigated and resolved.

\section{Ethics approval and consent to participate}

All experimental protocols were approved by the Ethics Committee of the First Affiliated Hospital of Xi'an Jiaotong University (Xi'an, China).

\section{Patient consent for publication}

Not applicable.

\section{Competing interests}

The authors declare that they have no competing interests.

\section{References}

1. Squadroni M, Tondulli L, Gatta G, Mosconi S, Beretta G and Labianca R: Cholangiocarcinoma. Crit Rev Oncol Hematol 116: 11-31, 2017

2. Razumilava $\mathrm{N}$ and Gores GJ: Cholangiocarcinoma. Lancet 383: 2168-2179, 2014

3. Blechacz B: Cholangiocarcinoma: Current knowledge and new developments. Gut Liver 11: 13-26, 2017.

4. Saha SK, Zhu AX, Fuchs CS and Brooks GA: Forty-year trends in cholangiocarcinoma Incidence in the U.S: Intrahepatic disease on the rise. Oncologist 21: 594-599, 2016.

5. Fairweather M, Balachandran VP and D'Angelica MI: Surgical management of biliary tract cancers. Chin Clin Oncol 5: 63, 2016.

6. Ghouri YA, Mian I and Blechacz B: Cancer review: Cholangiocarcinoma. J Carcinog 14: 1, 2015.

7. Xu J, Zhao $\mathrm{J}$ and Zhang R: Four microRNAs signature for survival prognosis in colon cancer using TCGA Data. Sci Rep 6: 38306, 2016.

8. Ge YZ, Wu R, Xin H, Zhu M, Lu TZ, Liu H, Xu Z, Yu P, Zhao YC, Li MH, et al: A tumor-specific microRNA signature predicts survival in clear cell renal cell carcinoma. J Cancer Res Clin Oncol 141: 1291-1299, 2015. 
9. Liang B, Li Y and Wang T: A three miRNAs signature predicts survival in cervical cancer using bioinformatics analysis. Sci Rep 7: 5624, 2017.

10. Puik JR, Meijer LL, Le Large TY, Prado MM, Frampton AE, Kazemier $\mathrm{G}$ and Giovannetti E: miRNA profiling for diagnosis, prognosis and stratification of cancer treatment in cholangiocarcinoma. Pharmacogenomics 18: 1343-1358, 2017.

11. Bartel DP: MicroRNAs: Genomics, biogenesis, mechanism, and function. Cell 116: 281-297, 2004.

12. Liu HT and Gao P: The roles of microRNAs related with progression and metastasis in human cancers. Tumour Biol: 2016 (Epub ahead of print).

13. Peng $\mathrm{Y}$ and Croce CM: The role of MicroRNAs in human cancer. Signal Transduct Target Ther 1: 15004, 2016.

14. Chandran UR, Medvedeva OP, Barmada MM, Blood PD Chakka A, Luthra S, Ferreira A, Wong KF, Lee AV, Zhang Z, et al: TCGA Expedition: A data acquisition and management system for TCGA Data. PLoS One 11: e0165395, 2016.

15. Robinson MD, McCarthy DJ and Smyth GK: edgeR: A Bioconductor package for differential expression analysis of digital gene expression data. Bioinformatics 26: 139-140, 2010.

16. Budczies J, Klauschen F, Sinn BV, Győrffy B, Schmitt WD, Darb-Esfahani S and Denkert C: Cutoff finder: A comprehensive and straightforward Web application enabling rapid biomarker cutoff optimization. PLoS One 7: e51862, 2012.

17. Zhang MY, Li SH, Huang GL, Lin GH, Shuang ZY, Lao XM, $\mathrm{Xu}$ L, Lin XJ, Wang HY and Li SP: Identification of a novel microRNA signature associated with intrahepatic cholangiocarcinoma (ICC) patient prognosis. BMC Cancer 15: 64, 2015.

18. Varkonyi-Gasic E, Wu R, Wood M, Walton EF and Hellens RP: Protocol: A highly sensitive RT-PCR method for detection and quantification of microRNAs. Plant Methods 3: 12, 2007.

19. Mitchell PS, Parkin RK, Kroh EM, Fritz BR, Wyman SK, Pogosova-Agadjanyan EL, Peterson A, Noteboom J, O'Briant KC, Allen A, et al: Circulating microRNAs as stable blood-based markers for cancer detection. Proc Natl Acad Sci USA 105: 10513-10518, 2008.

20. Wang Q, Tang H, Yin S and Dong C: Downregulation of microRNA-138 enhances the proliferation, migration and invasion of cholangiocarcinoma cells through the upregulation of RhoC/p-ERK/MMP-2/MMP-9. Oncol Rep 29: 2046-2052, 2013.

21. Zhu L, Huang F, Deng G, Nie W, Huang W, Xu H, Zheng S, Yi Z and Wan T: MicroRNA-212 targets FOXA1 and suppresses the proliferation and invasion of intrahepatic cholangiocarcinoma cells. Exp Ther Med 13: 2109, 2017.

22. Yamanaka S, Campbell NR, An F, Kuo SC, Potter JJ, Mezey E, Maitra A and Selaru FM: Coordinated effects of microRNA-494 induce $\mathrm{G}_{2} / \mathrm{M}$ arrest in human cholangiocarcinoma. Cell Cycle 11: 2729-2738, 2012.

23. Liu N, Jiang F, He TL, Zhang JK, Zhao J, Wang C, Jiang GX, Cao LP, Kang PC, Zhong XY, et al: The Roles of MicroRNA-122 overexpression in inhibiting proliferation and invasion and stimulating apoptosis of human cholangiocarcinoma cells. Sci Rep 5: 16566, 2015.

24. McNally ME, Collins A, Wojcik SE, Liu J, Henry JC, Jiang J, Schmittgen $\mathrm{T}$ and Bloomston M: Concomitant dysregulation of microRNAs miR-151-3p and miR-126 correlates with improved survival in resected cholangiocarcinoma. HPB (Oxford) 15: 260-264, 2013

25. Silakit R, Loilome W, Yongvanit $\mathrm{P}$, Chusorn $\mathrm{P}$, Techasen A, Boonmars T, Khuntikeo N, Chamadol N, Pairojkul C and Namwat N: Circulating miR-192 in liver fluke-associated cholangiocarcinoma patients: A prospective prognostic indicator. J Hepatobiliary Pancreat Sci 21: 864-872, 2014.

26. Wang LJ, Zhang KL, Zhang N, Ma XW, Yan SW, Cao DH and Shi SJ: Serum miR-26a as a diagnostic and prognostic biomarker in cholangiocarcinoma. Oncotarget 6: 18631-18640, 2015.

27. Li J, Gao B, Huang Z, Duan T, Li D, Zhang S, Zhao Y, Liu L, Wang Q, Chen Z and Cheng K: Prognostic significance of microRNA-203 in cholangiocarcinoma. Int J Clin Exp Pathol 8: 9512-9516, 2015.
28. Cheng Q, Feng F, Zhu L, Zheng Y, Luo X, Liu C, Yi B and Jiang X: Circulating miR-106a is a novel prognostic and lymph node metastasis indicator for cholangiocarcinoma. Sci Rep 5: $16103,2015$.

29. Deng $Y$ and Chen $Y$ : Increased expression of miR-29a and its prognostic significance in patients with cholangiocarcinoma. Oncol Res Treat 40: 128-132, 2017.

30. Du F, Yuan P, Zhao ZT, Yang Z, Wang T, Zhao JD, Luo Y, Ma F, Wang JY, Fan Y, et al: A miRNA-based signature predicts development of disease recurrence in HER2 positive breast cancer after adjuvant trastuzumab-based treatment. Sci Rep 6: 33825 , 2016.

31. Lu J, Getz G, Miska EA, Alvarez-Saavedra E, Lamb J, Peck D, Sweet-Cordero A, Ebert BL, Mak RH, Ferrando AA, et al: MicroRNA expression profiles classify human cancers. Nature 435: 834-838, 2005.

32. Fkih M'hamed I, Privat M, Ponelle F, Penault-Llorca F, Kenani A and Bignon YJ: Identification of miR-10b, miR-26a, miR-146a and miR-153 as potential triple-negative breast cancer biomarkers. Cell Oncol 38: 433-442, 2015.

33. Huang L, Lin JX, Yu YH, Zhang MY, Wang HY and Zheng M: Downregulation of six microRNAs is associated with advanced stage, lymph node metastasis and poor prognosis in small cell carcinoma of the cervix. PLoS One 7: e33762, 2012.

34. Wang J, Li Y, Ding M, Zhang H, Xu X and Tang J: Molecular mechanisms and clinical applications of miR-22 in regulating malignant progression in human cancer (Review). Int J Oncol 50: 345-355, 2017.

35. Xu D, Takeshita F, Hino Y, Fukunaga S, Kudo Y, Tamaki A, Matsunaga J, Takahashi RU, Takata T, Shimamoto A, et al: miR-22 represses cancer progression by inducing cellular senescence. J Cell Biol 193: 409-424, 2011.

36. Budd WT, Seashols-Williams SJ, Clark GC, Weaver D, Calvert V, Petricoin E, Dragoescu EA, O'Hanlon K and Zehner ZE: Dual action of miR-125b as a tumor suppressor and OncomiR-22 promotes prostate cancer tumorigenesis. PLoS One 10: e0142373, 2015.

37. Lin K, Xu T, He BS, Pan YQ, Sun HL, Peng HX, Hu XX and Wang SK: MicroRNA expression profiles predict progression and clinical outcome in lung adenocarcinoma. Onco Targets Ther 9: 5679-5692, 2016.

38. Song G, Zhang H, Chen C, Gong L, Chen B, Zhao S, Shi J, Xu J and Ye Z: miR-551b regulates epithelial-mesenchymal transition and metastasis of gastric cancer by inhibiting ERBB4 expression. Oncotarget 8: 45725-45735, 2017.

39. Yuan H, Chen Z, Bai S, Wei H, Wang Y, Ji R, Guo Q, Li Q, Ye Y, Wu J, et al: Molecular mechanisms of lncRNA SMARCC2/miR-551b-3p/TMPRSS4 ax is in gastric cancer. Cancer Lett 418: 84-96, 2018

40. Ewald F, Norz D, Grottke A, Hofmann BT, Nashan B and Jucker M: Dual inhibition of PI3K-AKT-mTOR- and RAF-MEK-ERK-signaling is synergistic in cholangiocarcinoma and reverses acquired resistance to MEK-inhibitors. Invest New Drugs 32: 1144-1154, 2014.

41. Guan L, Zhang L, Gong Z, Hou X, Xu Y, Feng X, Wang H and You $\mathrm{H}$ : FoxO3 inactivation promotes human cholangiocarcinoma tumorigenesis and chemoresistance through Keap1-Nrf2 signaling. Hepatology 63: 1914-1927, 2016.

42. Thongchot S, Yongvanit P, Loilome W, Seubwai W, Phunicom K, Tassaneeyakul W, Pairojkul C, Promkotra W, Techasen A and Namwat N: High expression of HIF-1alpha, BNIP3 and PI3KC3: Hypoxia-induced autophagy predicts cholangiocarcinoma survival and metastasis. Asian Pac J Cancer Prev 15: 5873-5878, 2014. 